\title{
Ciclos e Caminhos Longos em Grafos Ímpares*
}

\author{
Felipe de Campos Mesquita ${ }^{1}$ e Letícia Rodrigues Bueno ${ }^{1}$ \\ ${ }^{1}$ CMCC, Universidade Federal do ABC (UFABC), Santo André - SP - Brasil \\ felipe.mesquita@aluno.ufabc.edu.br, leticia.bueno@ufabc.edu.br
}

\begin{abstract}
The odd graph $O_{k}$ is the graph whose vertices are all subsets with $k$ elements of a set that has $n$ elements, and two vertices are joined by an edge if the corresponding pair of $k$-subsets is disjoint. A conjecture due to Biggs claims that $O_{k}$ is hamiltonian for $k \geq 3$ and a conjecture due to Lovász implies that $O_{k}$ has a hamiltonian path for $k \geq 1$. From a hamiltonian cycle in $O_{k-1}$, we show how to construct a cycle in $O_{k}$ with at least $75 \%$ of the vertices of $O_{k}$. Additionally, we prove that, for each $k$, the odd graph has a path with at least $50 \%$ of the vertices of $O_{k}$.
\end{abstract}

Resumo. O grafo ímpar $O_{k}$ é o grafo cujos vértices são todos os subconjuntos de tamanho $k$ de um conjunto com $(2 k+1)$ elementos e dois vértices são adjacentes se eles são disjuntos. Uma conjectura atribuída a Biggs afirma que o grafo $O_{k}$ é hamiltoniano para $k \geq 3$ e uma conjectura atribuída a Lovász implica que $O_{k}$ tem um caminho hamiltoniano para $k \geq 1$. A partir de um ciclo hamiltoniano em $O_{k-1}$, mostramos como construir um ciclo em $O_{k}$ com pelo menos $75 \%$ dos vértices de $O_{k}$. Adicionalmente, nós provamos que, para todo $k$, o grafo ímpar tem um caminho com pelo menos $50 \%$ dos vértices de $O_{k}$.

\section{Introdução}

Dado um grafo $G=(V(G), E(G))$, um caminho hamiltoniano é um caminho que passa por todos os vértices de $G$ (Figura 1(a)). Um ciclo hamiltoniano é um ciclo que passa por todos os vértices de $G$ (Figura 1(b)). Um grafo é hamiltoniano se tem um ciclo hamiltoniano.

O problema do ciclo hamiltoniano consiste em determinar se um grafo é hamiltoniano e foi provado ser NP-Completo [Karp 1972]. O problema do caminho hamiltoniano consiste em determinar se um grafo tem um caminho hamiltoniano e também é conhecido ser NP-Completo [Garey and Johnson 1979]. Devido à dificuldade inerente aos problemas NP-Completos, neste trabalho procuramos resolver os problemas de ciclos e caminhos hamiltonianos restritos à classe de grafos ímpares, com o objetivo de encontrar uma solução para os problemas propostos através das propriedades estruturais desta classe de grafos.

O grafo ímpar ("odd graph", em inglês), denotado por $O_{k}$, tem como seu conjunto de vértices todos os subconjuntos de tamanho $k$ tomados do conjunto $\{1,2, \ldots, n=2 k+$ $1\}$. Dois subconjuntos são adjacentes se eles são disjuntos. Veja na Figura 1 exemplos de grafos ímpares.

\footnotetext{
${ }^{*}$ Esse trabalho foi financiado pelo CNPq.
} 


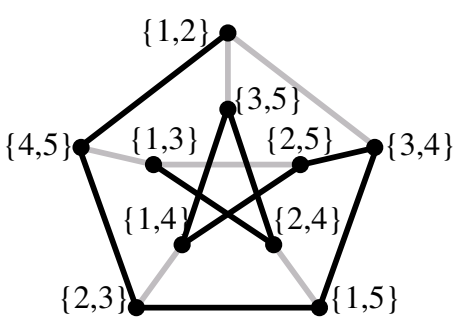

(a) $\mathrm{O}_{2}$, onde $k=2$ e $n=5$

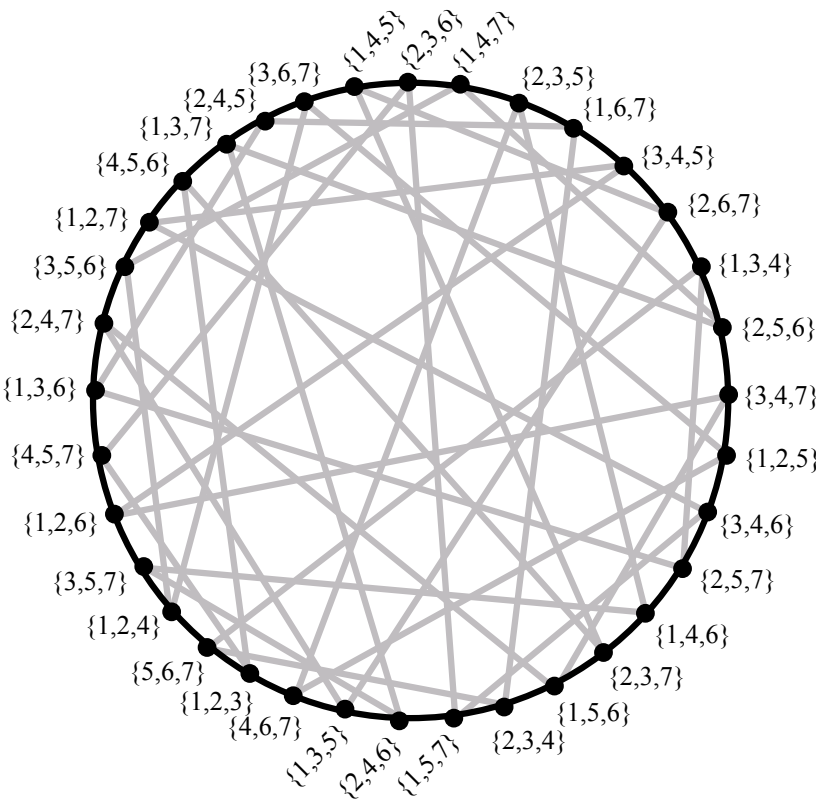

(b) $O_{3}$, onde $k=3$ e $n=7$

Figura 1. Os grafos ímpares para $k=2,3$. Em (a) um caminho hamiltoniano em destaque. Em (b) um ciclo hamiltoniano em destaque.

Os grafos ímpares têm sido estudados por muitos pesquisadores, especialmente pelo seu alto grau de simetria. De fato, os problemas de ciclos e caminhos hamiltonianos em grafos ímpares são fortemente motivados pelas conjecturas de Lovász [Lovász 1970] e Biggs [Biggs 1979]:

Conjectura 1 (Lovász, 1970). Todo grafo não-direcionado, conexo e vértice-transitivo tem um caminho hamiltoniano.

Conjectura 2 (Biggs, 1979). O grafo ímpar $O_{k}$ é hamiltoniano para todo $k>2$.

Desde que os grafos ímpares são vértice-transitivos, podem fornecer um contraexemplo para a Conjectura de Lovász. No entanto, o grafo $O_{k}$ tem $\left(\begin{array}{c}2 k+1 \\ k\end{array}\right)$ vértices (veja Tabela 1), o que impede uma computação exata de caminhos e ciclos hamiltonianos em $O_{k}$ para grandes valores de $k$. Através de um algoritmo heurístico, foi mostrado que os grafos ímpares são hamiltonianos para $k \leq 13$ [Shields and Savage 2004], com exceção do grafo $\mathrm{O}_{2}$ que é o grafo de Petersen e que já foi provado não ser hamiltoniano, embora tenha um caminho hamiltoniano (Figura 1 (a)). Posteriormente, foi provado que os grafos ímpares tem um caminho hamiltoniano para $k \leq 17$ [Bueno et al. 2009].

Em relação a ciclos ou caminhos longos, foi provado que existe uma constante $c$ tal que, para todo $k, O_{k}$ tem um ciclo de comprimento de pelo menos $(1-$ $\left.\frac{c}{\sqrt{k}}\right)\left|V\left(O_{k}\right)\right|$ [Johnson 2004]. A prova não tenta estimar $c$ mas, em outras palavras, significa que o grafo $O_{k}$ é assintoticamente hamiltoniano pois, conforme $k$ aumenta, o comprimento do ciclo também aumenta.

Em nosso trabalho, procuramos resolver a Conjectura 2 através de uma construção recursiva que determine um ciclo (ou caminho) hamiltoniano em $O_{k}$ a partir de um ciclo (ou caminho) hamiltoniano em $O_{k-1}$. Neste objetivo, até agora, conseguimos um ciclo (respectivamente, caminho) em $O_{k}$ com pelo menos $75 \%$ de seus vértices, a partir de um 
Tabela 1. Número de vértices de alguns grafos ímpares

\begin{tabular}{|c|c|c|}
\hline$k$ & $n=2 k+1$ & $\left|V\left(O_{k}\right)\right|=\left(\begin{array}{c}2 k+1 \\
k\end{array}\right)$ \\
\hline 1 & 3 & 3 \\
\hline 2 & 5 & 10 \\
\hline 3 & 7 & 35 \\
\hline 4 & 9 & 126 \\
\hline 5 & 11 & 462 \\
\hline 6 & 13 & 1.716 \\
\hline 7 & 15 & 6.435 \\
\hline 8 & 17 & 24.310 \\
\hline 9 & 19 & 92.378 \\
\hline 10 & 21 & 352.716 \\
\hline 11 & 23 & 1.352 .078 \\
\hline 12 & 25 & 5.200 .300 \\
\hline 13 & 27 & 20.058 .300 \\
\hline 14 & 29 & 77.558 .760 \\
\hline 15 & 31 & 300.540 .195 \\
\hline 16 & 33 & 1.166 .803 .110 \\
\hline 17 & 35 & 4.537 .567 .650 \\
\hline
\end{tabular}

ciclo (respectivamente, caminho) hamiltoniano em $O_{k-1}$ (Teorema 1).

Ainda em busca de uma prova para a Conjectura 2, encontramos que todo grafo ímpar tem um caminho com pelo menos $50 \%$ de seus vértices (Teorema 2).

\section{Resultados}

A seguir, mostramos como construir um ciclo em $O_{k}$ com pelo menos $75 \%$ dos vértices de $O_{k}$. Para isso, utilizamos um ciclo hamiltoniano em $O_{k-1}$. Antes, porém, precisamos definir alguns conceitos que serão utilizados na prova do resultado.

Considere a correspondência entre os $k$-subconjuntos e os $(k+1)$-subconjuntos de $\{1,2, \ldots, 2 k+1\}$ com um conjunto de strings binárias de $n$ bits com exatamente $k$ 1 's e $(k+1) 0$ 's. A correspondência $b_{n} b_{n-1} \ldots b_{1} \rightarrow\left\{i \mid b_{i}=1\right\}$ é uma bijeção de strings binárias de $n$ bits para os subconjuntos de $n=2 k+1$. Veja o grafo $O_{2}$ (Figura 1 (a)) nesta representação na Figura 2.

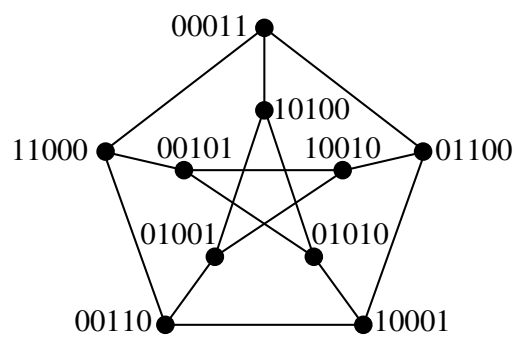

Figura 2. $\mathrm{O}$ grafo $\mathrm{O}_{2}$ com vértices representados como strings binárias. 
Construímos dois caminhos disjuntos em $O_{k}$ a partir de um caminho ou ciclo hamiltoniano $C=\left(v_{1}, v_{2}, v_{3}, v_{4} \ldots, v_{q}\right)$ em $O_{k-1}$. Considere os vértices de $C$ representados como strings binárias. Ao acrescentarmos 1 e 0 a um vértice de $C$ obtemos, por definição, um vértice de $O_{k}$. Assim, construímos um caminho $C_{1}$ em $O_{k}$ como segue: $C_{1}=\left(0 v_{1} 1,1 v_{2} 0,0 v_{3} 1,1 v_{4} 0, \ldots, 1 v_{q} 0\right)$ se $q$ é par e $C_{1}=\left(0 v_{1} 1,1 v_{2} 0,0 v_{3} 1,1 v_{4} 0, \ldots, 0 v_{q} 1\right)$ se $q$ é ímpar. Similarmente, construímos um caminho $C_{2}=\left(1 v_{1} 0,0 v_{2} 1,1 v_{3} 0,0 v_{4} 1, \ldots, 0 v_{q} 1\right)$ se $q$ é par e $C_{2}=$ $\left(1 v_{1} 0,0 v_{2} 1,1 v_{3} 0,0 v_{4} 1, \ldots, 1 v_{q} 0\right)$ se $q$ é ímpar. Note, pela construção, que $C_{1}$ e $C_{2}$ são caminhos em $O_{k}$ e, se $q$ é par, ambos são também ciclos.

Seja $S$ o conjunto de vértices de $O_{k}$ tal que, para todo $v \in S, v=0 v^{\prime} 1$ ou $v=1 v^{\prime} 0$ onde $v^{\prime} \in V\left(O_{k-1}\right)$.

Lema 1. $|S|=\left|C_{1}\right|+\left|C_{2}\right|$.

Demonstração. Sem perda de generalidade, assuma $v=0 v^{\prime} 1$, onde $v \in S$ e $v^{\prime} \in$ $V\left(O_{k-1}\right)$. Então $v^{\prime}$ precisa conter os demais $(k-1) 1$ 's. Estes podem ser colocados em $\left(\begin{array}{c}2 k-1 \\ k-1\end{array}\right)$ combinações possíveis nos bits restantes de $v$, ou seja, são exatamente os vértices de $O_{k-1}$. O caso é análogo para $v=1 v^{\prime} 0$. Pela construção de $C_{1}$ e $C_{2}$, todo vértice de $S$ está ou em $C_{1}$ ou em $C_{2}$.

Os vértices de $O_{k}$ que não estão em $S$ são: (a) $0 w^{\prime} 0$ onde $w^{\prime} \in\left(\begin{array}{c}n-2 \\ k\end{array}\right)=\left(\begin{array}{c}2 k-1 \\ k\end{array}\right)$, e; (b) $1 w^{\prime \prime} 1$ onde $w^{\prime \prime} \in\left(\begin{array}{c}n-2 \\ k-2\end{array}\right)=\left(\begin{array}{c}2 k-1 \\ k-2\end{array}\right)$. Denote por $T$ o conjunto dos vértices $0 w^{\prime} 0$, $w^{\prime} \in\left(\begin{array}{c}2 k-1 \\ k\end{array}\right)$. Pela relação das combinações complementares obtemos:

$$
\begin{gathered}
\left(\begin{array}{c}
2 k-1 \\
k
\end{array}\right)=\left(\begin{array}{c}
2 k-1 \\
(2 k-1)-k
\end{array}\right) \\
\left(\begin{array}{c}
2 k-1 \\
k
\end{array}\right)=\left(\begin{array}{c}
2 k-1 \\
k-1
\end{array}\right) \\
|T|=\left|V\left(O_{k-1}\right)\right|
\end{gathered}
$$

Teorema 1. Se existe um ciclo (respectivamente, caminho) hamiltoniano $C=$ $\left(v_{1}, v_{2}, \ldots, v_{q}\right)$ em $O_{k-1}$, então $O_{k}$ tem um ciclo (respectivamente, caminho) $C^{\prime}$ tal que $\left|C^{\prime}\right| \geq 0.75\left|V\left(O_{k}\right)\right|$.

Demonstração. Primeiro, assuma que $C$ é um ciclo hamiltoniano. Mostramos agora que existem $q$ vértices $0 \overline{v_{j}} 0$ conectando $0 v_{j} 1$ a $1 v_{j} 0$, onde $0 v_{j} 1 \in S, 1 v_{j} 0 \in S$ e $v_{j} \in C$ para $1 \leq j \leq q$. Antes, porém, observe que todo vértice $v_{j} \in C$ tem $k 1$ 's e $(k-1) 0$ 's em seu complemento $\overline{v_{j}}$. Assim, $0 \overline{v_{j}} 0 \in V\left(O_{k}\right)$. Construímos agora $q$ caminhos de tamanho 3 combinando os vértices de $C_{1}, C_{2}$ e $T$ :

$$
\begin{gathered}
Q_{1}=0 v_{1} 1,0 \overline{v_{1}} 0,1 v_{1} 0 \\
Q_{2}=1 v_{2} 0,0 \overline{v_{2}} 0,0 v_{2} 1 \\
\vdots \\
Q_{q}=1 v_{q} 0,0 \overline{v_{q}} 0,0 v_{q} 1 \text {, se } q \text { é par ou } \\
Q_{q}=0 v_{q} 1,0 \overline{v_{q}} 0,1 v_{q} 0, \text { se } q \text { é ímpar. }
\end{gathered}
$$


Observe que, para $Q_{j}, 1 \leq j \leq q$, o primeiro vértice de $Q_{j}$ está em $C_{1}$, o segundo vértice está em $T$ e o terceiro vértice está em $C_{2}$. Sabemos que os $q$ vértices $0 \overline{v_{j}} 0$, para $v_{j} \in C$, são distintos, desde que $C$ é um ciclo hamiltoniano em $O_{k-1}$.

Denotamos por $Q^{\prime} \circ Q^{\prime \prime}$ o caminho obtido por percorrer os vértices de um caminho $Q^{\prime}$ seguidos pelos vértices de um caminho $Q^{\prime \prime}$. Dado um caminho $Q^{\prime}$, denotamos por $\overleftarrow{Q^{\prime}}$ o caminho atravessado do último para o primeiro vértice.

Concatenamos os $q$ caminhos $Q_{j}, 1 \leq j \leq q$, como segue:

$C^{\prime}=Q_{1} \circ \overleftarrow{Q}_{2} \circ Q_{3} \circ \overleftarrow{Q}_{4} \circ \ldots \circ \overleftarrow{Q}_{q}$, se $q$ é par e

$C^{\prime}=Q_{1} \circ \overleftarrow{Q}_{2} \circ Q_{3} \circ \overleftarrow{Q}_{4} \circ \ldots \circ Q_{q}$, se $q$ é ímpar

As Figuras 3 e 4 ilustram a construção de $C^{\prime}$ para $q$ par e $q$ ímpar, respectivamente.

Note que, em ambos os casos, o último vértice de $C^{\prime}$ é $1 v_{q} 0$. Como o primeiro vértice de $C^{\prime}$ é $0 v_{1} 1$, segue que $C^{\prime}$ é um ciclo, já que $v_{1}$ e $v_{q}$ são adjacentes no ciclo $C$. Assuma agora que $C$ é um caminho hamiltoniano. Então $C^{\prime}$ é somente um caminho, pois o vértice $0 v_{1} 1$ não é adjacente à $1 v_{q} 0$, já que $v_{1}$ não é adjacente a $v_{q}$ em $C$.

Mostramos agora que $\left|C^{\prime}\right|=\left|C_{1}\right|+\left|C_{2}\right|+|T| \geq 0.75\left|V\left(O_{k}\right)\right|$ :

$$
\begin{array}{ccc}
3\left|V\left(O_{k-1}\right)\right|>0.75\left|V\left(O_{k}\right)\right| & > \\
\left|V\left(O_{k}\right)\right| & <4\left|V\left(O_{k-1}\right)\right| \\
\frac{\left|V\left(O_{k}\right)\right|}{\left|V\left(O_{k-1}\right)\right|} & < & 4 .
\end{array}
$$

Temos $\begin{aligned} &\left|V\left(O_{k-1}\right)\right|=\left(\begin{array}{c}2 k-1 \\ k-1\end{array}\right)=\frac{(2 k-1) !}{k !(k-1) !} \mathrm{e} \\ &\left|V\left(O_{k}\right)\right|=\left(\begin{array}{c}2 k+1 \\ k\end{array}\right)=\frac{(2 k+1) !}{(k+1) ! k !}=\frac{(2 k+1)(2 k)(2 k-1) !}{(k+1)(k)(k-1) ! k !} .\end{aligned}$

Segue que $\left|V\left(O_{k}\right)\right|=\frac{(2 k+1)(2 k)}{(k+1)(k)}\left|V\left(O_{k-1}\right)\right|$

$$
\frac{\left|V\left(O_{k}\right)\right|}{\left|V\left(O_{k-1}\right)\right|}=\frac{(2 k+1)(2 k)}{(k+1)(k)} \quad=\frac{(4 k+2)}{(k+1)} \text {. }
$$

Verificamos que:

$$
\lim _{k \rightarrow \infty} \frac{\left|V\left(O_{k}\right)\right|}{\left|V\left(O_{k-1}\right)\right|}=\lim _{k \rightarrow \infty} \frac{(4 k+2)}{(k+1)}=\lim _{k \rightarrow \infty}\left(4-\frac{2}{k+1}\right)=4 .
$$

Das expressões (1) e (2), temos:

$$
\begin{array}{lcc}
\frac{(4 k+2)}{(k+1)} & \leq & 4 \\
\frac{4(k+1)-2}{k+1} & = & 4-\frac{2}{k+1} \leq 4 \\
\frac{-2}{(k+1)} & \leq & 0 .
\end{array}
$$

Logo, para $k>0$, temos que $\left|C^{\prime}\right|=3\left|V\left(O_{k-1}\right)\right| \geq 0.75\left|V\left(O_{k}\right)\right|$.

Pelo Lema 1 e Teorema 1, $C^{\prime}$ contém todos os vértices de $S$ e $T$. Assim, para que $C^{\prime}$ seja um ciclo ou caminho hamiltoniano em $O_{k}$, é preciso acrescentar a $C^{\prime}$ os vértices $1 w^{\prime \prime} 1$ onde $w^{\prime \prime} \in\left(\begin{array}{c}2 k-1 \\ k-2\end{array}\right)$. 


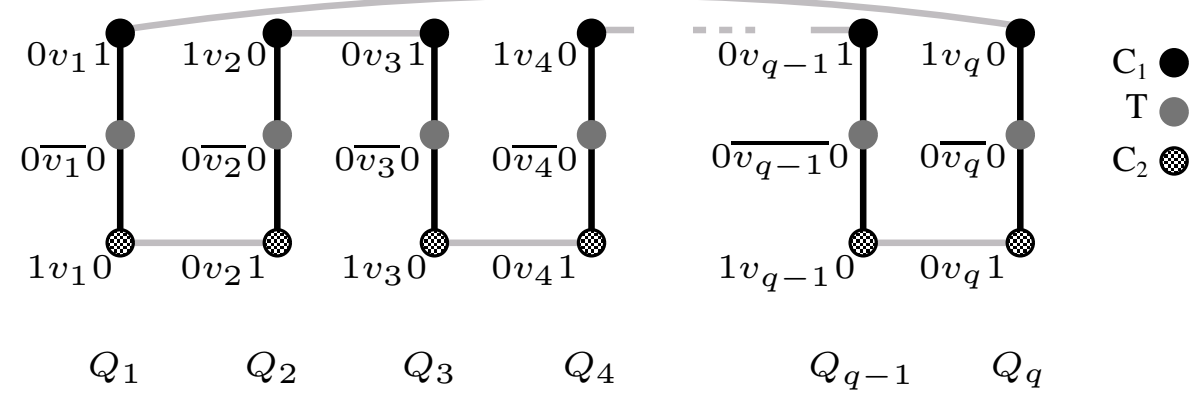

Figura 3. Teorema 1: construção de $C^{\prime}$ para $q$ par. Os $q$ caminhos de tamanho 3 (arestas em negrito) são concatenados pelas arestas de $C_{1}$ e $C_{2}$ (arestas em cinza).

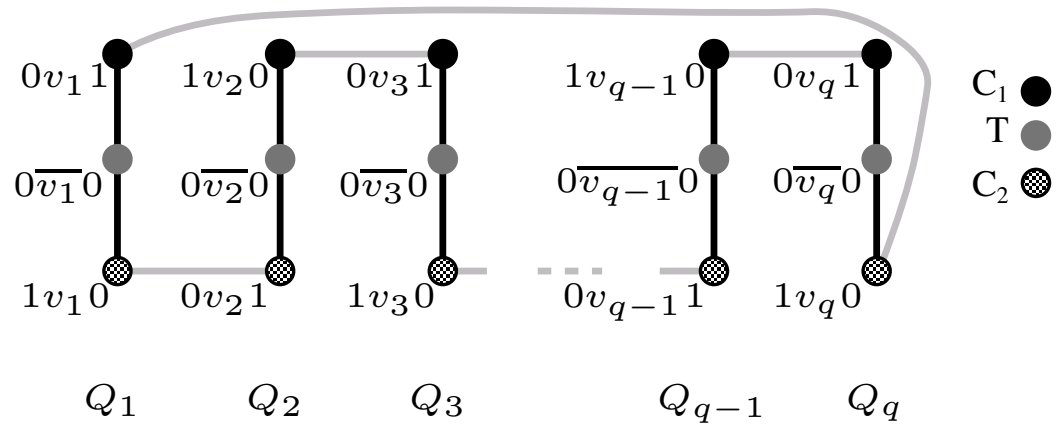

Figura 4. Teorema 1: construção de $C^{\prime}$ para $q$ ímpar. Os $q$ caminhos de tamanho 3 (arestas em negrito) são concatenados pelas arestas de $C_{1}$ e $C_{2}$ (arestas em cinza).

Mostramos, a seguir, que o grafo $O_{k}$ tem um caminho de comprimento maior ou igual a $50 \%$ dos vértices do grafo. Antes, porém, definimos alguns conceitos utilizados na prova deste resultado.

O grafo $n$-cubo, denotado por $Q_{n}$, tem como vértices uma sequência de $n$ bits, e dois vértices são adjacentes se eles diferem em exatamente um bit. $\mathrm{O} n$-cubo pode ser representado pelo diagrama de Hasse do reticulado booleano de ordem $n$ (veja Figura 5).

O código Gray binário [Gilbert 1958, Gray 1953] é uma enumeração de todas as strings binárias tal que os elementos diferem em exatamente um bit. Defina o peso de uma string como o número de 1's na string. O código Gray monotônico [Savage and Winkler 1995] é um código Gray no qual pares consecutivos de strings de pesos $i, i+1$ precedem os pares de strings de pesos $j, j+1$ para todo $i<j$. Veja um exemplo na Figura 5.

Teorema 2. Para $k \geq 1$, o grafo ímpar $O_{k}$ tem um caminho $P$ tal que $|P| \geq 0.5\left|V\left(O_{k}\right)\right|$. Demonstração. Seja $p^{\prime}$ um código Gray monotônico em $Q_{n}$ e seja $e_{n}(i)$ o número de arestas unindo o nível $i$ ao nível $i+1$ de $p^{\prime}$, onde um nível $i$ contém os vértices com $i$ 1's (Figura 5). Então, para cada $i, 0 \leq i \leq n-1, p^{\prime}$ contém um caminho de comprimento $e_{n}(i)+1$ no subgrafo formado pelos vértices dos níveis $i$ e $i+1$. Considere agora o $(n-1)$-cubo $Q_{n-1}$. Seja $P$ o caminho de um código Gray monotônico contido no subgrafo formado pelos níveis $k$ e $(k+1)$. Denote por $P^{\prime}$ o caminho $P$ com um bit 0 a esquerda de cada string binária e cada string binária $w$ de peso $(k+1)$ substituída por 


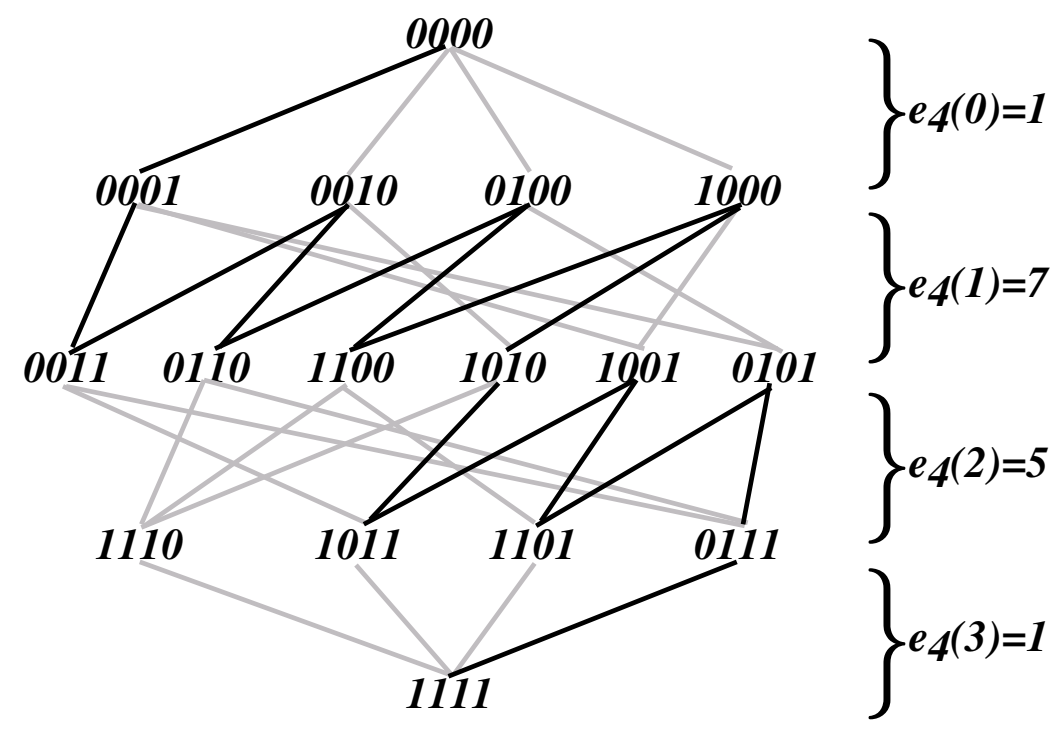

Figura 5. O cubo $Q_{4}$ no diagrama de Hasse com um código Gray monotônico em destaque e o número de arestas do código Gray monotônico por nível.

$\bar{w}$. Observe que $P^{\prime}$ é um caminho em $O_{k}$ pois o código Gray monotônico não repete vértices e nenhum deles contém o elemento $2 k+1$, já que $P$ está em $Q_{4}$. Precisamos agora determinar o comprimento de $P^{\prime}$. De acordo com [Savage and Winkler 1995], $e_{n}(i)$ pode ser determinada pela seguinte recorrência:

$$
\begin{aligned}
& e_{n}(0)=1 \\
& e_{n}(1)=2\left(\begin{array}{c}
n \\
i
\end{array}\right)-e_{n}(i-1) \text { para } 1 \leq i \leq n-1 \text { cuja solução é } \\
& e_{n}(i)=2\left(\begin{array}{c}
n-1 \\
i
\end{array}\right)-(-1)^{i} .
\end{aligned}
$$

Assim, o comprimento de $P^{\prime}$ em $Q_{n-1}$ em relação ao número de vértices de $O_{k}$ é:

$$
\begin{aligned}
\frac{\left|P^{\prime}\right|}{\left|V\left(O_{k}\right)\right|}=\frac{e_{n-1}(k)+1}{\left(\begin{array}{l}
n \\
k
\end{array}\right)} & =\frac{2\left(\begin{array}{c}
n-2 \\
k
\end{array}\right)-(-1)^{k}+1}{\left(\begin{array}{l}
n \\
k
\end{array}\right)} \\
& =\frac{\left(2\left(\begin{array}{c}
2 k-1 \\
k
\end{array}\right)-(-1)^{k}\right)+1}{\left(\begin{array}{c}
2 k+1 \\
k
\end{array}\right)} \\
& =\frac{2 \cdot\left(\frac{(2 k-1) !}{k !(k-1) !}\right)-(-1)^{k}+1}{\frac{(2 k+1) !}{k !(k+1) !}} \\
& =2 \cdot \frac{(2 k-1) !}{(k-1) !} \cdot \frac{(k+1) !}{(2 k+1) !} \\
& =\frac{2 \cdot(2 k-1) !(k+1) \cdot k \cdot(k-1) !}{(k-1) !(2 k+1)(2 k)(2 k-1) !} \\
& =\frac{(k+1)}{(2 k+1)} \geq \frac{1}{2} .
\end{aligned}
$$


Como um exemplo para o Teorema 2, considere o grafo $Q_{4}$ (Figura 5). Determinamos um caminho $P=(1010,1011,1001,1101,0101,0111)$ em $O_{2}$ no grafo $Q_{4}$. Acrescentamos um bit 0 a esquerda de cada string binária, obtendo (01010, 01011, 01001, 01101, 00101, 00111) e substituímos as strings de peso $(k+1)$ por seu complemento: $P^{\prime}=(01010,10100,01001,10010,00101,11000) \in O_{k}$. Então $P^{\prime}$ é um caminho no grafo $\mathrm{O}_{2}$ (Figura 2) e $\left|P^{\prime}\right|=6>5=0.5\left|V\left(\mathrm{O}_{2}\right)\right|$.

\section{Conclusões e Trabalhos Futuros}

Em nosso trabalho, mostramos como, a partir um ciclo hamiltoniano em $O_{k-1}$, construir um ciclo em $O_{k}$ com pelo menos $75 \%$ dos vértices de $O_{k}$ (Teorema 1). Se $O_{k-1}$ tem somente um caminho hamiltoniano, então obtemos um caminho hamiltoniano em $O_{k}$ com pelo menos $75 \%$ dos vértices de $O_{k}$. Desde que os grafos ímpares tem um ciclo hamiltoniano para $3 \leq k \leq 13$ [Shields and Savage 2004] e um caminho hamiltoniano para $k \leq 17$ [Bueno et al. 2009], o Teorema 1 implica na existência de um ciclo em $O_{14}$ com $75 \%$ dos vértices do grafo e de um caminho em $O_{18}$ com $75 \%$ dos vértices do grafo. Um caminho hamiltoniano já é conhecido para $O_{14}$ [Bueno et al. 2009], mas para o grafo $O_{18}$ este é o primeiro resultado obtido até o momento, em nosso melhor conhecimento.

Para $k$ geral, nós mostramos que o grafo $O_{k}$ tem um caminho $P$ tal que $|P| \geq$ $0.5\left|V\left(O_{k}\right)\right|$ (Teorema 2). Com exceção do trabalho de [Johnson 2004] que implica que os grafos ímpares são assintoticamente hamiltonianos, nosso resultado é a única aproximação de caminhos e ciclos longos para os grafos ímpares para $k$ geral até o momento.

Como trabalho futuro, pretendemos melhorar o Teorema 1 a fim de obter $100 \%$ dos vértices no ciclo construído, respondendo assim a conjectura de Biggs. Por outro lado, melhorar o Teorema 2 provendo ciclos e/ou caminhos mais longos resulta em uma aproximação melhor para problemas desafiadores como os propostos pelas conjecturas de Lovász e Biggs. Portanto, pretendemos trabalhar também no Teorema 2.

Ciclos e caminhos hamiltonianos são também muito estudados em uma classe de grafos conhecida como grafos $B_{k}$ ou grafos ímpares duplos ("doubled odd graphs", em inglês) que são formados a partir dos grafos ímpares e são também vértice-transitivos. Havel [Havel 1983] conjecturou que os grafos $B_{k}$ são hamiltonianos para $k \geq 1$. Devido à relação entre as duas classes de grafos, a resolução da conjectura de Biggs [Biggs 1979] automaticamente forneceria ciclos hamiltonianos para o grafo $B_{k}$ para todo $k$ ímpar, resolvendo parcialmente a conjectura de Havel.

Relativo à contribuição do aluno, destacamos que os resultados deste trabalho foram obtidos pelo próprio aluno que, desde o início do projeto, já contava com boa fundamentação matemática. O texto foi escrito pelo aluno sob supervisão da orientadora e os trabalhos futuros propostos serão trabalhados no próximo projeto de Iniciação Científica. A orientadora contribuiu com: sua experiência e conhecimento no problema, possíveis direções para a pesquisa, instruções de como formalizar e organizar os resultados em forma de lemas e teoremas, supervisão do trabalho e verificação da corretude dos resultados.

\section{Referências}

Biggs, N. (1979). Some odd graph theory. Ann. New York Acad. Sci., 319:71-81.

Bueno, L. R., Faria, L., Figueiredo, C. M. H., and Fonseca, G. D. (2009). Hamiltonian paths in odd graphs. Appl. Anal. Discrete Math., 3(2):386-394. 
Garey, M. R. and Johnson, D. S. (1979). Computers and Intractability, A Guide to the Theory of NP-Completeness. W.H. Freeman and Company, New York.

Gilbert, E. N. (1958). Gray codes and paths on the n-cube. Bell Systems Tech. J., 37:815826.

Gray, F. (1953). Pulse code communication. U.S. Patent 2632058.

Havel, I. (1983). Semipaths in directed cubes. In Fiedler, M., editor, Graphs and other Combinatorial Topics, pages 101-108, Teubner, Leipzig. Teubner-Texte Math.

Johnson, J. R. (2004). Long cycles in the middle two layers of the discrete cube. $J$. Combin. Theory Ser. A, 105(2):255-271.

Karp, R. M. (1972). Reducibility among combinatorial problems. In Miller, R. and Thatcher, J., editors, Complexity of Computer Computations, pages 85-103, New York. Plenum Press.

Lovász, L. (1970). Problem 11. In Combinatorial Structures and their Applications. Gordon and Breach.

Savage, C. D. and Winkler, P. (1995). Monotone gray codes and the middle levels problem. J. Combin. Theory Ser. A, 70(2):230-248.

Shields, I. and Savage, C. D. (2004). A note on hamilton cycles in Kneser graphs. Bulletin of the Institute for Combinatorics and Its Applications, 40:13-22. 